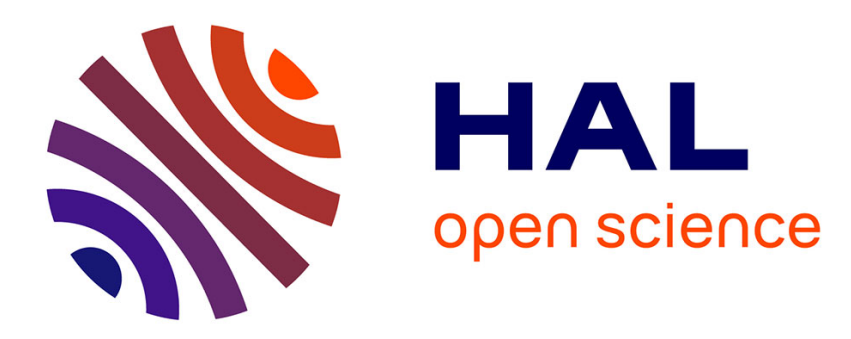

\title{
Mythologie centralienne, mythologie polytechnicienne
}

Christian Hottin

\section{To cite this version:}

Christian Hottin. Mythologie centralienne, mythologie polytechnicienne. Livraisons d'Histoire de l'Architecture, 2001, n 1, 1er semestre 2001, p. 61-75. halshs-00086814

\section{HAL Id: halshs-00086814 https://shs.hal.science/halshs-00086814}

Submitted on 19 Jul 2006

HAL is a multi-disciplinary open access archive for the deposit and dissemination of scientific research documents, whether they are published or not. The documents may come from teaching and research institutions in France or abroad, or from public or private research centers.
L'archive ouverte pluridisciplinaire HAL, est destinée au dépôt et à la diffusion de documents scientifiques de niveau recherche, publiés ou non, émanant des établissements d'enseignement et de recherche français ou étrangers, des laboratoires publics ou privés. 


\section{MYTHOLOGIE CENTRALIENNE, MYTHOLOGIE POLYTECHNICIENNE}

ChristianHOTTIN

Mission ethnologie

Direction de l'architecture et du patrimoine

Christian.hottin@culture.gouv.fr

"Polytechnique : rêve de toutes les mères [...]. Fureur du bourgeois dans les émeutes quand il apprend que l'École polytechnique sympathise avec les ouvriers ! (...)" ${ }^{1}$. Comme en écho au savoureux poncif de Flaubert, Maurice Donnay, académicien et centralien écrit : "En 1830, [les Centraliens] combattent avec autant d'élan que ceux de Polytechnique, ça se voit moins, parce qu'ils n'ont pas d'uniforme"2. Il y a dans le propos de Donnay une pointe d'ironie, un peu d'amertume aussi. Le fait est que Polytechnique seule fait partie des mythes de la Nation, et que cette dimension de l'institution est intégrée par les membres de la communauté polytechnicienne aux représentations du groupe ${ }^{3}$. Mais si l'École polytechnique est porteuse de représentations exogènes, les deux établissements ont secrété, tout au long de leur histoire, des représentations endogènes, qui tendent à former, à travers les textes, les oeuvres d'art ou l'architecture des bâtiments, une image de l'école. Parmi toutes les facettes possibles de ces portraits narcissiques, deux retiennent ici l'attention. Comment met-on en scène la naissance de l'institution ? Comment se construit et se décrit le rapport de la communauté aux lieux qui l'abritent ? Choisir de suivre pas à pas ces deux écoles dans la construction de leurs personnalités respectives invite à porter son attention tantôt sur l'une, tantôt sur l'autre : la spécificité de chaque discours se manifeste alors avec plus d'évidence.

\footnotetext{
${ }^{1}$ Gustave Flaubert, Le Dictionnaire des idées reçues, texte établi présenté et annoté par Anne Herschberg Pierrot, Paris, Le Livre de poche, 1997, 251 p., p. 74.

${ }^{2}$ Maurice Donnay, Centrale, Paris, Nouvelle société d'édition, 1930, 137 p., p. 30.

${ }^{3}$ "Mythal : qui a trait au mythe de l'École. Le bicorne est un objet mythal. Le défilé du 14 juillet est un moment mythal. Danser le quadrille en GU [grand uniforme] au bal de l'X est une activité éminemment mythale. L'utilisation de la tangente [épée] pour sabrer une bouteille de champagne également ». Fabrice Mattiata, Dictionnaire d'Argot de l’X, École polytechnique, Palaiseau, 1994, 141 p., p. 90.
} 


\section{HISTOIRE ET SOCIOLOGIE DE DEUX INSTITUTIONS}

Préambule obligé d'une approche comparée des mythologies propres aux deux institutions, le simple récit chronologique des grandes étapes de leur histoire depuis leur fondation jusqu'à nos jours offre lui-même un dialogue riche d'enseignements.

Tout comme l'École normale ou l'École des langues orientales vivantes, l'École polytechnique est l'œuvre de la Convention, qui l'institua sous le nom d'École centrale des travaux publics. Elle eut alors son siège au Palais Bourbon. C’est Napoléon I ${ }^{\text {er }}$ qui lui donna sa physionomie pratiquement définitive en 1806, lorsque le jeune établissement, nouvellement doté d'un statut militaire, s'installa sur la Montagne Sainte-Geneviève dans les bâtiments occupés avant la Révolution par les collèges de Navarre et de Boncourt. Dès cette époque, la mission de l'institution était de former en deux ans des jeunes gens recrutés sur concours et destinés à entrer par la suite dans des écoles d'application civiles ou militaires. Le service de l'État, sous une forme ou sous une autre, était donc le débouché naturel des études à Polytechnique. En 1829 naquit l'École centrale des arts et manufactures, marquée par les pratiques du libéralisme anglo-saxon et par le développement en France de la Révolution industrielle. Alphonse Lavallée, maitre d'œuvre de cette entreprise, dut s'endetter pour lancer le projet. La jeune école s’installa dans un quartier industrieux, non loin du Conservatoire des arts et métiers, en louant les bâtiments de l'ancien hôtel de Juigné. Issues de projets pédagogiques et de philosophies fort différents, les deux écoles scientifiques prirent place dans des locaux d'Ancien régime. Rien d'étonnant à cela, puisque avant 1847, date de l'installation de l'École normale dans le cloître de la rue d’Ulm imaginé par Alphonse de Gisors, aucune grande école parisienne ne vivait dans un cadre spécialement conçu pour elle. En 1857, Centrale fut cédée à l'État après des négociations qui établirent, entre autres dispositions, que l'institution restait libre d'utiliser directement ses bénéfices pour augmenter ses collections ou développer les études en son $\operatorname{sein}^{4}$.

Alors que, depuis la Restauration, l'École polytechnique, appartenant au groupe des édifices entretenus par la Direction des bâtiment civils et des palais nationaux, n'avait eu de cesse de faire son "pré carré", en réaménageant les locaux existants, en en détruisant d’autres, ou en recherchant l'expropriation des maisons mitoyennes, les dirigeants de Centrale, voyant arriver à expiration le bail de l'hôtel de la rue de Thorigny, obtinrent de faire construire un bâtiment neuf, juste derrière le Conservatoire des arts et métiers, sur le site de l'ancien marché Saint-Martin.

\footnotetext{
4 Projet de Loi relatif à la cession de l'École centrale des arts et manufactures, Lille, impr. A. David, 1857, 13 p.
} 
L'édifice fut occupé à partir de $1884^{5}$. On voit d'emblée que, si les raisons de ces transformations sont semblables (augmentation progressive du nombre des élèves et diversification des enseignements), les moyens mis en oeuvre pour moderniser l'établissement diffèrent totalement.

De part et d'autre de la Grande Guerre, qui frappe lourdement les deux établissements, les centenaires de 1894 et 1929 donnent lieu à des cérémonies grandioses, étalées sur plusieurs jours et longuement préparées, au cours desquelles les institutions, avec un narcissisme évident, contemplent leurs succès passés et dressent le bilan de leur action. Le rayonnement international de ces festivités, grâce aux invitations envoyées et aux télégrammes reçus, leur donne une image de leur position au sein de la société en tant qu'organismes voués à la production des élites et à la reproduction de leurs comportements.

Jusqu'en 1960 la question toujours lancinante du manque de place est résolue par la construction et la reconstruction de locaux sur le site (à Polytechnique), ou par l'aménagement des sous-sols et l'établissement d'annexes dans le XII ${ }^{e}$ arrondissement (à Centrale). La volonté politique de décentralisation des grandes écoles, qu'elle soit réelle ou qu’il s'agisse de rumeurs insistantes, semble avoir joué un rôle décisif, à l'aube de la Ve République, pour mettre en branle une réflexion sur le déménagement ${ }^{6}$. Tandis que l'École centrale s'installe en 1969 à Châtenay-Malabry, l'École polytechnique quitte en 1976 la rue Descartes pour le plateau de Saclay, sur la commune de Palaiseau?

Quant à la sociologie de ces deux institutions, elle présente de nombreux points communs : sur ce plan, les différences sont probablement moins importantes aujourd'hui qu'au siècle dernier. Les modes de recrutement ont été unifiés, toutes deux recrutant essentiellement sur concours à l'issue de l'année de mathématiques spéciales. Par le passé, les préparations aux concours (la "piste" et la "taupe") étaient en effet distinctes. Comme dans les autres grandes écoles d'ingénieurs, les élèves sont issus majoritairement des couches aisées de la population. En revanche, si les stratégies de carrière lancées à la fin des études permettent dans les deux cas d'espérer atteindre des postes de direction très rémunérateurs, elles n'en sont pas moins différentes. Certes, la place des Polytechniciens au sein de l'Armée a fortement diminué. En revanche, le passage par les écoles d'application fait que les premières années de la vie

\footnotetext{
${ }^{5}$ Voir : Christian Hottin, «La maison des abeilles : l'Ecole Centrale des Arts et Manufactures rue Montgolfier et rue Conté (1889-1969) », Le Paris de Centraliens, bâtisseurs et entrepreneurs, Jean-François Belhoste (dir.), Paris, AAVP, 2004, 236 p., p. 43-48. Texte également disponible sur HAL-SHS.

${ }^{6}$ Voir : Christian Hottin, "Les délices du campus ou le douloureux exil : trois grandes écoles parisiennes face à leur transfert (1950 -1980) ", L'arcbitecture scolaire, essai d'bistoriographie internationale (Anne-Marie Châtelet et Marc Le Cœur dir.) numéro spécial de la Revue d'bistoire de l'éducation, $\mathrm{n}^{\circ}$ 102, mai 2004), p. 267 - 293. Texte également disponible sur HAL-SHS.

${ }^{7}$ Les bâtiments de Centrale sont depuis occupés par une annexe du Conservatoire national des arts et métiers, ceux de Polytechnique on été partagés entre le Ministère de l'enseignement supérieur et le Collège de France, tandis qu'un petit local, l'ancienne entrée principale ou « boite à claque », était attribué à l'association des anciens élèves.
} 
professionnelle du Polytechnicien se passent au sein de la fonction publique, les départs dans le secteur privé étant ensuite très nombreux. Il n'en va pas de même à Centrale, où les élèves ne sont pas fonctionnaires et où les liens avec les entreprises sont structurellement beaucoup plus forts. Ce rapport privilégié au monde des entreprises existe depuis l'origine de l'institution, et même si le phénomène est peut-être moins sensible de nos jours, on a eu raison de remarquer que Centrale attirait volontiers des élèves moins nantis scolairement que ceux de Polytechnique, mais dotés d'un capital social (par exemple des parents appartenant au monde du patronat) qui leur permettait de mieux tirer parti de leur titre ${ }^{8}$. Par ces derniers aspects, le recrutement des Centraliens offre des points communs avec celui des élèves d'HEC, de même que les deux écoles offrent des ressemblances dans leur histoire institutionnelle?

\section{LE RÉCIT DES ORIGINES}

Dans les deux écoles, une importance particulière est attachée aux conditions dans lesquelles furent fondées les institutions. Ce moment de la fondation n'est pas seulement celui de la création matérielle de l'école, avec les premiers professeurs, la première promotion, et l'installation dans les locaux. Tel qu’il apparaît dans les récits élaborés ultérieurement par les historiens des deux écoles (souvent d'anciens élèves ${ }^{10}$ ), ce moment est celui qui ramasse en une action conduite par quelques hommes exceptionnels toutes les caractéristiques, toutes les vertus de l'époque. La création est toujours une expérience fondatrice résolument nouvelle (les précédentes fondations sont passées sous silence, ou voient leur importance minimisée), et elle est présentée comme le commencement d'une geste qui se poursuit encore sous nos yeux. Ainsi, la fondation de Centrale est toujours montrée dans les ouvrages de la même manière, selon un credo qui n'est guère remis en doute d'un livre à l'autre : la revendication de la filiation anglo-saxonne, les écoles d'ingénieurs anglaises ayant servi de modèle à la création de 1829, la conception totalement moderne de l'École, éventuellement en passant en revue les écoles existantes, de façon à expliquer qu'elles ne ressortissent pas au même modèle et qu'elles sont trop

\footnotetext{
${ }^{8}$ Pierre Bourdieu, La Noblesse d'État, grandes écoles et esprit de corps, Paris, éd. de Minuit, 1989, 569 p., p. 219. Pierre Bourdieu renvoie pour plus de détails à : J.H. Weiss, The Making of Technological Man : the Social Origins of French Engineering Education, Cambridge, Mass., MIT Press, 1982.

${ }^{9}$ HEC, comme Centrale, a partie liée dès son origine avec les milieux d'affaires et constitue une réponse à leur désir de posséder une institution de formation de cadres indépendante de l'université. Pierre Bourdieu, La Noblesse État, Paris, éd. de Minuit, 1989, 569 p., p. 279.

${ }^{10}$ Maurice Donnay, Centrale, Paris, Nouvelle société d'édition, 1930, 137 p., p. 19-32.
} 
étroitement liées à l'État, et enfin la volonté de se démarquer des écoles qui, en France, sous la Restauration, sont les plus proches du projet de l'École centrale, à savoir les écoles des Arts et Métiers ${ }^{11}$. Dans cette perspective, la mise en scène quasiment hagiographique des fondateurs occupe une place centrale, y compris dans les monographies les plus récentes : "Ce furent quatre jeunes gens n'ayant à mettre que de faibles ressources matérielles dans la balance du destin, qui, réunis, devinrent les apôtres de cette nouvelle croyance" ${ }^{\prime 12}$. Quelques années avant Patrice Noailles, les auteurs du livre publié à l'occasion du cent-cinquantenaire de la fondation usaient d'un ton assez semblable pour qualifier les initiateurs du projet : "Faisant un retour en arrière, nous pouvons être fiers de l'béritage laissépar nos anciens et, en premier lieu, par nos fondateurs. Il leur fallut une singulière imagination, et un courage hors du commun, pour créer une école d'ingénieurs à l'époque où ils le firent, car en ce milieu de 1829, ni la situation politique, ni le système éducatif français n'incitaient à prendre un tel risque" ${ }^{\prime 13}$. On apporte une attention toute particulière à la personnalité des ces fondateurs, venus d'horizon différents et dont la diversité des profils est délibérément mise en avant, comme un gage de l'originalité et de la nouveauté de l'Institution. L'énumération de leurs noms s'impose comme une figure rituelle du discours sur l'institution, quel qu'en soit le vecteur : texte, peinture ou sculpture. Les bustes des quatre fondateurs étaient disposés sur les paliers du grand escalier de l'École centrale rue Montgolfier et leurs portraits ornent encore aujourd'hui le vestibule du pavillon de l'administration à Châtenay-Malabry.

Les fondateurs de Polytechnique ont également droit à l'image : Lagrange, Berthollet, Fourcroy, Laplace et Monge sont sculptés en bas-relief au-dessus de la porte de la "boite à claque", le pavillon d'entrée des élèves ${ }^{14}$. Accompagnés de Lazare Carnot et Lamblardie, on les retrouve peints en médaillons par Colin ${ }^{15}$, leurs noms figurent sur les murs ${ }^{16}$ de certains bâtiments et les bustes de plusieurs d'entre eux sont installés dans la bibliothèque ${ }^{17}$, puis transportés dans le grand hall de Palaiseau. Enfin, les fondateurs réunis en séance figurent en bonne place sur l'immense

\footnotetext{
${ }^{11}$ Un exemple parmi d'autres : Patrice Noailles, École centrale de Paris, Paris, 1984, 158 p., p. 20-21.

${ }^{12}$ Discours prononcé par le directeur de l'école, M. Cauvet, Inauguration de la nouvelle École centrale, 5 novembre 1884, Paris, Imprimerie nouvelle, 1885, 11 p., p. 5.

13 École centrale (1829-1979), Paris, 1979, 156 p., p. 8.

${ }^{14}$ Archives de l'École polytechnique, Palaiseau, titre V, section 3, carton 4.

15 Arch. nat., F 21128.

${ }^{16}$ Ceux de Monge, Lamblardie et Carnot sont inscrits à l'angle de la rue d'Arras parmi des emblèmes civils et militaires. L'Illustration, Ier semestre 1875, 17 avril 1875, p. 255.

${ }^{17}$ Ce sont les bustes de Fourcroy, Monge et Lamblardie.
} 
toile de Dupain, l'Apothéose de l'École polytechnique, véritable condensé des mythes de l'institution. Le récit des origines de l'École polytechnique est cependant plus complexe que celui de Centrale. Chez les polytechniciens, comme dans d'autres établissements (par exemple l'École des chartes), différents moments apparaissent investis d'une valeur fondatrice : la création par la Convention, incontestablement, est l'un d'eux. Mais la période napoléonienne n'est pas moins importante. C'est alors que se produisent certains des événements, réels ou supposés, qui, repris et mis en forme par les historiographes de l'institution, contribueront à la légende dorée de l'établissement et à la construction du Polytechnicien idéal. On en veut pour exemple l'escarmouche qui oppose, à la fin de la campagne de France, une batterie tenue par des élèves aux troupes russes de Barclay : l'épisode sera ensuite magnifié et fondu en bronze sous la forme du Conscrit de Theunissen, puis édité en modèle réduit et vendu en série. Plus riche encore d'implications est le mot prêté à l'empereur toujours en 1814 (“Je ne suis pas encore réduit à tuer ma poule aux oenfs d'or"). L'image de la Poule (l'école) couvant les précieux enfants (les élèves) de l'Empire (puis de la Nation, grâce à l'assimilation aisée de cette dernière au coq gaulois) a connu une grande fortune iconographique : on la retrouve au frontispice d'un ouvrage ${ }^{18}$, au plafond de la bibliothèque, sur la verrière de l'amphithéâtre Arago, dans la salle des conseils et sur les murs ${ }^{19}$. En outre, à Polytechnique, le prestige de l'emplacement choisi par Napoléon pour établir l'institution invite les auteurs à faire remonter la filiation encore plus haut dans le temps. Le rapport entre la fondation révolutionnaire et le Collège de Navarre, création royale, n'est pas explicitement mis au service de la construction du discours sur l'École Polytechnique. En revanche, certaines études, publiées par des auteurs issus de l'École, font une large part au prestige de la création capétienne, et font abondamment l'éloge de la qualité de son enseignement ${ }^{20}$. Il est extrêmement tentant de voir dans ce texte l'affirmation d'un rapport de filiation qui inscrit l'établissement dans une généalogie ancienne et prestigieuse : "La Montagne Sainte-Geneviève est un de ces lieux saints de l'intelligence qui suscitent en certains points du monde une ferveur mystique. l'École polytechnique continue d'entretenir la flamme sacrée allumée sur la docte montagne six siècles plus tôt ${ }^{\prime 21}$. A propos de l'agrandissement des locaux, le poète dit :

"Sur le mont qui jadis au temps théologal

De Navarre abrita le studieux mystère

\footnotetext{
${ }^{18}$ Le Livre du Centenaire, Paris, 1894. Illustration reprise dans : Le Paris des Polytechniciens, catalogue de l'exposition organisée par l'Action Artistique de la Ville de Paris, Paris, 1994, 299 p., p. 110.

${ }^{19}$ Paul Truffau, Livre d'or de l'École polytechnique, Paris et Casablanca, 1962, 187 p., p. 31.

${ }^{20}$ Gaston Pinet, «Le Collège de Navarre et l'École polytechnique », La Grande revue, 10 juillet 1909, p. 66-89.

${ }^{21}$ M. Prévost, Polytechnique, Paris, Nouvelle société d'édition, Paris, 1931, 123 p., p. 16.
} 


\section{Notre École a quitté son vieil air monacal \\ Et resplendit de frais accueil et de lumière." 22}

Le changement est ici revendiqué, avec le passage des anciens locaux, encore marqués par leur passé clérical, mais le rapport de parenté et la continuité entre les deux établissements n'en sont pas moins mentionnés.

Onction de l'État révolutionnaire et impérial d'une part, idéal libéral de l'école conçue et fondée comme une entreprise de l'autre : les représentations de l'institution à l'État sont affectés par ces schémas initiaux. Au rapport fort et cependant ambigu vis à vis de la puissance publique qui se manifeste dans les discours des Polytechniciens, s'oppose l'attitude de réserve et presque de défiance qui transparaît dans les récits des Centraliens. La cession de l'École en 1857 est un épisode manifestement délicat de l'histoire de l'établissement, présenté de manière détaillée, en insistant sur le fait qu'il fut librement consenti par les dirigeants de l'époque, et que cet "accord à l'amiable" ne lésait pas les intérêts centraliens, puisque Centrale conservait une grande autonomie de gestion. Les textes produits au moment de la construction, et surtout lors de l'inauguration des bâtiments de la rue Montgolfier, sont révélateurs de la tension qui oppose les instances dirigeantes de Centrale aux pouvoirs publics. : retards, difficultés, problèmes techniques sont systématiquement imputés à la ville, accusée de subventionner trop chichement le projet ${ }^{23}$, et à l'administration des Beaux-Arts, coupable aux yeux des dirigeants de lenteur dans l'examen du dossier ${ }^{24}$. Et un auteur, de conclure une fois le bâtiment terminé : "L'école a largement appliqué le proverbe "Aide toi et le ciel t'aidera", elle s'est aidée comme aucun établissement d'instruction ne l'avait encore fait en France. Nous espérons qu'il lui en sera tenu compte"25. On retrouve ailleurs cette attitude distante vis à vis de l'État, et ce désir affiché de se passer de ses services dans la mesure du possible : les décors peints et sculptés de l'École, à la différence de ceux présents dans les autres institutions d'enseignement supérieur, ont été financés presque exclusivement par les membres de la communauté, on ne trouve pas de commandes de l'État, seulement quelques rares subventions

\footnotetext{
22 «Transformations de Paris», Le Figaro Illustré, février 1931, p. 61-62. Les vers signés « P.H. » sont attribués à un élève.

${ }^{23}$ François. Pothier, dans un livre publié peu après l'achèvement des travaux va plus loin, et fait le compte des richesses apportées par l'École à Paris depuis 1829 (évaluées à 50 millions de francs) du simple fait de son existence pour critiquer la mesquinerie de la municipalité. François. Pothier, Histoire de l'École centrale des arts et manufactures, Paris, 1887, 554 p., p. 363.

${ }^{24}$ Charles de Comberousse, «L'École centrale des arts et manufactures 1829-1882 », La nouvelle revue, t. 28, 1882, p. 51-85.

${ }^{25}$ Charles de Comberousse, «L'École centrale », Le Magasin pittoresque, 1886, 54e année, p. 347-350.
} 
dont le caractère honorifique est souligné par les Centraliens ${ }^{26}$.

Fort différentes dans leur rapport à l'État, les deux écoles le sont aussi dans les relations qu'elles entretiennent avec leurs locaux.

\section{LA MAISON COMMUNE}

Les classes préparatoires et les grandes écoles en général peuvent être définies comme des institutions qui exercent sur leurs membres une forme d" enfermement symbolique" 27 ; l'analyse sociologique met en effet en évidence les facteurs qui favorisent le repli de la communauté sur elle-même et le développement d'un esprit clanique : la charge de travail à fournir, la spécificité des matières enseignées, l'intériorisation des règles de conduite non écrites qui régissent non seulement la vie quotidienne mais aussi la construction des projets liés à la vie professionnelle. Il faut y ajouter, dans le cas de Polytechnique ou de Normale, l'internat ou le casernement des élèves $^{28}$. De même, le développement des argots spécifiques est un phénomène parfaitement illustratif de cet enfermement ${ }^{29}$. L'étude des champs sémantiques couverts par ces idiomes particuliers est un bon point de départ pour une approche différente de cette situation, tendant à montrer non plus ses implications sur le comportement, mais la manière dont l'espace de l'enfermement est vécu et approprié. Or, une part très importante du vocabulaire formé par les élèves est précisément constituée de mots désignant les lieux, les bâtiments, où les objets de la vie quotidienne. Autant d'usages qui concourent à faire de l'espace commun un espace saturé de significations, puissamment investi d'un sens spécifique, celui qui lui est conféré par le groupe ${ }^{30}$. La langue est un des aspects essentiels de la construction de la maison commune, mais pas le seul.

Dans les deux établissements, le trait le plus frappant de cette appropriation des lieux réside sans doute dans l'accumulation des oeuvres peintes ou sculptées et des monuments

\footnotetext{
26 Arch. nat. , F 21 4849, dossier de l'École centrale des arts et manufactures.

27 Pierre Bourdieu, La Noblesse d'État, grandes écoles et esprit de corps, Paris, éd. de Minuit, 1989, 569 p., p. 121-131. Voir aussi, p. 176-181, «Quelques documents sur l'expérience de la vie dans les classes préparatoires et les grandes écoles ». Les témoignages portent cependant principalement sur les comportements vécus en classe préparatoire.

${ }^{28}$ Cette dernière remarque ne s'applique pas, dans un premier temps, à Centrale, qui ne connaît au XIXe siècle que le régime de l'externat. La situation change lors de la création, en 1928, d'une maison destinée aux étudiants. Gabriel Morice, «La maison des élèves de l'École centrale », L'Architecture, vol. XLI, n5, 1928, p. 151-154.

${ }^{29}$ Pierre Bourdieu, La noblesse d'État, Paris, éd. de Minuit, 1989, 569 p., p. 122,

${ }^{30}$ Claudine Billoux, «L'X : le Mythe, l'enfermement et la fuite », Universités et grandes écoles à Paris, Paris, A.A.V.P., 1999, 222 p., p. 169-173.
} 
épigraphiques qui sont dispersés à travers toute l'école ${ }^{31}$. On a déjà fait mention, à Centrale, des bustes des fondateurs exposés dans les niches de l'escalier d'honneur. Au pied des marches se trouve encore aujourd'hui le monument aux Centraliens morts pour la France ou victimes du devoir professionnel, oeuvre de Denys Puech ${ }^{32}$. Non loin de là, formant porte d'entrée pour les élèves, se dresse l'autre monument aux morts, dédiés aux Centraliens morts en 1914-1918 ${ }^{33}$. Le monument a été dessiné par Pierre Leprince-Ringuet et sculpté par Marcel Gaumont. Dans le réfectoire des élèves ce sont des fresques de Leprince-Ringuet, un ancien élève, qui comparent les activités des ingénieurs aux attributs des dieux de l'Olympe. Au premier étage, dans les salons de la direction, des peintures plus anciennes de Pierre Vauthier mettent en scène les élèves eux-mêmes au cours de leurs travaux pratiques. Pour Polytechnique, dont les bâtiments furent occupés pendant près de 170 ans, les oeuvres sont beaucoup plus nombreuses : les sculptures de Romagnési sur le portail d'entrée, les bas-reliefs disposés de part et d'autre de l'entrée de l'amphithéâtre Poincaré, les statues des maréchaux Joffre et Foch, le Conscrit de Theunissen dans la cour du pavillon Boncourt, ou encore les peintures du foyer des élèves ${ }^{34}$. Dans un cas comme dans l'autre, il n'est pas une de ces oeuvres qui ne recèle, de par son sujet ou son iconographie, une part du discours constitutif de l'identité du groupe, qu'il s'agisse de son histoire ou de ses caractéristiques supposées. Certaines sont à leur tour sources de discours et génératrices d'identité.

S'agissant de l'École polytechnique et de l'École centrale de la rue Montgolfier, l'énumération des espaces nécessaires à la vie des deux institutions fait apparaittre d'évidentes similitudes, en dépit de la spécificité militaire de Polytechnique : salles de cours, amphithéâtres, salles d'études, bibliothèque, laboratoires. Ces deux programmes sont pourtant contenus dans des enveloppes architecturales très différentes, ne serait-ce qu'en raison de l'opposition fondamentale qui existe entre un édifice ancien, qui a fait l'objet d'une appropriation difficile ${ }^{35}$ et une

\footnotetext{
${ }^{31}$ S'agissant des locaux parisiens, le phénomène évoqué ici fait référence à un état antérieur aux années 1970 . Il est devenu difficilement perceptible pour le visiteur, les oeuvres ayant été transportées en banlieue à l'exception des plus volumineuses, tels que les monuments aux morts.

32 Sur cette sculpture : Arch. nat. F 21 4849, dossier de l'École centrale, subventions. Voir aussi : Denys Puech (1854-1942), Paris, 1993, 257 p., p. 125.

33 «L'Arche symbolique de l'École centrale », L'Illustration, 2eme semestre 1923, 10 novembre 1923, p. 465.

34 Les bas-reliefs sont toujours en place, les statues se trouvent à Palaiseau. Les «fresques» ont disparu. Reproduction : Paul Truffeau, Livre d'or de l'École polytechnique, Paris et Casablanca, 1962, 160 p., p. 100.

35 Sur l'histoire des bâtiments de Polytechnique : Werner Szambien, «Les bâtiments de la Montagne Sainte-Geneviève au XIXe siècle », Le Paris des Polytechniciens, des ingénieurs dans la ville, 1794-1994, Paris, DAAVP, 1994, p. 27-37.
} 
construction totalement neuve bâtie selon une programme mis au point grâce à la concertation des architectes et des enseignants ${ }^{36}$. Cette différence dans le rapport originel de l'institution à ses bâtiments n'est lui-même qu'un élément parmi d'autres de la richesse des représentations particulières de chaque école avec les lieux qui l'abritent.

Si les bâtiments occupés pendant près de deux siècles par Polytechnique sont véritablement l'espace propre à la communauté polytechnicienne, c'est peut-être plus par un long travail de sédimentation, fait d'usages et de souvenirs, que par l'action des architectes des bâtiments civils qui, deux siècles durant, se sont efforcés de construire des prolongements en harmonie avec l'élégante élévation du pavillon des bacheliers de Navarre. Ce long corps de bâtiment à trois niveaux coiffé d'une mansarde, cette façade lisse, à peine animée de quelques décrochements organisés autour du fronton triangulaire qui en marque le centre a constitué, pour ainsi dire, la matrice de l'architecture de l'école. Seul le pavillon de l'administration, ancien Collège de Boncourt, relève d'une esthétique différente. Partout ailleurs, la marque de l'architecture française classique est sensible sur les façades. Toutes les transformations imposées par le développement de l'institution s'inscrivent dans cette tradition, y compris la destruction pure et simple du bâtiment des bacheliers et son remplacement par un cube de béton : le fronton est seul conservé ${ }^{37}$ ! Tandis que de nouveaux locaux apparaissaient sans cesse, les constructions anciennes étaient vouées à la destruction : ainsi disparurent l'ancienne chapelle et la bibliothèque de Navarre. Dans cet espace en mue perpétuelle, ce sont les usages humains qui, jusque dans leur discontinuité et leur renouvellement, façonnent une école conforme à l'esprit des élèves. Il faut revenir sur l'usage de l'argot : par lui le lieu acquiert l'esprit de l'École, par la dénomination spécifique qui lui est attribuée il échappe à son statut générique pour devenir un attribut unique de la vie polytechnicienne : plus de cabinets d'aisances, mais des "longchamps", plus de tabouret, mais un "bouret", pas de store, mais un "zurlin". De même, une architecture peut être détournée de sa fonction première pour prendre une place singulière dans la géographie spécifique de l'institution : la sculpture ornementale du bélier, point de passage obligé pour les escapades nocturnes devient le "bêta", et tous les endroits susceptibles d'offrir un service identique reçoivent ce nom, qui devient alors commun. La formation des termes argotiques est un objet d'étude en soi. Par rapport au problème présent de la relation au lieu, il faut souligner que l'objet ou l'édifice deviennent polytechniciens, par apocope ou aphérèse (voir le bouret déjà cité, mais aussi les

\footnotetext{
${ }^{36}$ Sur les bâtiments de Centrale à Paris : Laurent Cascail, Mathieu Graton, Arnaud Grojean, «L'École Centrale, rue Montgolfier », Universités et grandes écoles à Paris, Paris, AAVP, 1999, 222 p., p. 133-138.

37 «Inauguration du bâtiment Joffre à l'École polytechnique », France Illustration, janvier-juin 1950, p. 446. Cet avatar malheureux a disparu à son tour, après le départ de l'École, au profit d'une nouvelle façade qui s'inspire de celle détruite précédemment...
} 
binets et les amphis), par métaphore ou métonymie (on va aux longchamps comme on irait aux courses), mais que ces divers procédés ont encore plus d'efficacité lorsque le terme choisi renvoie lui-même à l'histoire de l'établissement ou du groupe : les stores furent mis en place à l'instigation du colonel Zurlinden et l'horloge de la cour, dite "Berzee", tire son nom d'une légende propre à la communauté $^{38}$. L'appropriation de l'architecture n'est pas uniquement l'œuvre de la longue durée : l'événement est toujours propice à la célébration conjointe de l'esprit de l'école et de la grandeur de ses bâtiments. D’autres vers que ceux déjà cités en témoignent :

\author{
"Rendons grâce aux amis dont l'effort tutélaire \\ Féconde ce projet, à vous, mon général, \\ A ta fougue Umbdenstock, à ton calme, Tournaire, \\ A ta science rude, art obsidional \\ $[\cdots]$ \\ Honneur à toi d'avoir pour garder nos façades \\ Déconcerté l'assaut des fantômes maussades \\ Et paré de soleil les travaux de nos gars" ${ }^{39}$
}

Le corollaire de l'usage par lequel on s'approprie un lieu est la remémoration, la commémoration de cet usage et sa cristallisation sous une forme pérenne, pour ainsi dire académique telle que l'entrée dans un dictionnaire, lorsqu'il a été remplacé par de nouvelles pratiques. La littérature produite par les membres du groupe est au cœur de cette deuxième phase de sédimentation. Trois livres consacrés à l'argot de Polytechnique ont été publiés depuis que l’École existe ${ }^{40}$, le dernier ne date que de $1994^{41}$. La fonction mémorielle des ces ouvrages est explicitement mentionnée dans certaines préfaces : parcourir le livre, c'est revoir les lieux de l'école, se rappeler les actions accomplies au sein de cet espace, par quel moyen et de quelle façon ${ }^{42}$. Hors des dictionnaires d'argot, l'identification de l'école à ses objets ou à ses lieux spécifiques peut survenir dans un contexte plus large, ainsi Gaston Claris écrit : “le bouret pour nous c'est l'école”. Puis il évoque la boite

\footnotetext{
${ }^{38}$ Claudine Billoux, «L’X : le Mythe, l'enfermement et la fuite », Universités et grandes écoles à Paris, Paris, A.A.V.P., 1999, 222 p., p. 171.

39 «Transformations de Paris », Le Figaro illustré, février 1931, p. 61. La deuxième strophe rend hommage au procédé employé, le stick B (un type de revêtement des pierres).

40 Le plus ancien et le plus célèbre : Albert-Lévy et G.aston Pinet, L'Argot de l’X, illustré par les X, Paris, Alphonse Picard et Khan, 1894, XIII-326 p.

${ }^{41}$ Fabrice Mattiata, Dictionnaire d'Argot de l'X, École polytechnique, Palaiseau, 1994, 141 p.

42 R. Smet, Le nouvel argot de l’X, Paris, Gauthier-Villars, 1936, XIV-306 p..
} 
à claque et dit "sentir son cour battre plus vivement"

De 1829 à 1884, l'École centrale vécut dans les murs de l'hôtel de Juigné. Après cinquante-cinq ans passés dans des locaux qui ne lui appartenaient pas, elle s'installa dans un bâtiment neuf. Cet édifice fut conçu spécifiquement en fonction des besoins de l'établissement. De fait, le rapport entre l'école et son bâtiment s'établit d'une manière toute différente de celle observée à Polytechnique : si le travail de sédimentation existe également, il n'a pas la même valeur ni la même force. En revanche, le programme de construction constitue à lui seul une vision programmée de l'institution, un manifeste de son identité et de ses aspirations. L'édifice intègre dans son architecture les références historiques fondatrices de Centrale : le grand escalier qui conduit à l'administration est la réplique de celui de l'hôtel de Juigné, tandis que le parti décoratif choisi pour les façades, des murs de briques avec parements et bossages d'angles en pierre, sans être très original, n'en constitue pas moins un rappel possible des élévations du Conservatoire des arts et métiers voisin. On songe même un temps à renforcer l'identité centralienne du quadrilatère retenu pour site de la nouvelle école en donnant aux rues qui le délimitent les noms des fondateurs. Les élévations indiquent la destination des différentes ailes : la façade orientale, plus monumentale, dominée par un avant-corps couronné d'un fronton triangulaire, est celle des bureaux de l'administration. Sur les autres côtés, le métal est apparent, les croisées plus larges : ce sont les salles d'études, les amphithéâtres. Lors de l'inauguration le parti architectural est célébré comme étant celui qui convient parfaitement à la destination de l'édifice : “Cette préoccupation, visible chez. l'architecte, d'exprimer par le monument même le caractère de l'enseignement auquel il sert est la marque d'un puissant esprit. Nulle recherche du clinquant, nul désir de sacrifier au public par un étalage de combinaisons architecturales plus brillantes que raisonnées, Partout au contraire une science approfondie de l'art de bâtir, un ingénieux emploi de la pierre, de la brique et du fer, une entente merveilleuse des besoins auxquels doivent répondre les différents services de l'École" ${ }^{44}$. On évoque également à propos de l'édifice "un aspect manufacturier [...] Ces fenêtres plus larges que hautes, par lesquelles la lumière entre à flots, ces pilastres en maçonnerie de brique mêlées de sommiers de pierre dure servant d'appui aux fers des linteaux [qui] rappellent les grandes constructions industrielles des filatures $d u$ Nord ${ }^{45}$. La disposition intérieure des services répond, elle aussi, parfaitement aux besoins de l'école : chaque étage est affecté à une promotion, avec les bureaux en dépendant, les salles d'études des élèves et l'amphithéâtre, installé dans un pavillon d'angle. L'École centrale donne donc son architecture pour centralienne. Elle en est un manifeste. Ceci

\footnotetext{
${ }^{43}$ Gaston Claris, Notre École polytechnique, Paris, 1895 , XII-410 p., p. 2-3.

44 «La nouvelle École centrale », LTllustration, 18 octobre 1884, 2e semestre 1884, p. 247.

45 «La nouvelle École centrale », LTllustration, 18 octobre 1884, 2e semestre 1884, p. 253.
} 
est d'autant plus sensible que les textes, discours, articles ou récits historiques insistent tous sur le fait que les concepteurs du bâtiment sont eux-mêmes des Centraliens : Denfer et Demiduid. La nouvelle école, inaugurée en 1969 par Georges Pompidou est elle aussi l'œuvre de quatre architectes qui "sont tous des camarades" ${ }^{46}$ : Vitale, Drouin, Demaret et Fayeton". L’École centrale est faite par et pour les centraliens. C'est une affaire de famille, comme le révèle le cérémonial complexe de la pose de la première pierre à Châtenay-Malabry en 1965 : le major de troisième année lit le parchemin qui porte les grandes dates de l'histoire de l'École, le texte est déposé dans un tube métallique avec les médailles de l'établissement. Le tube est glissé dans une alvéole de la première pierre. Le ministre scelle la pierre avec le major de deuxième année, tandis que celui de première année tient l'auge à mortier ${ }^{48}$. L'École centrale est faite par et pour les centraliens, avec l'argent des centraliens : aussi souvent que possible, lorsque l'occasion se présente, on fait appel à l'autofinancement. Ce trait se rencontre également à Polytechnique, mais, pour autant que les sources le laissent supposer, il ne fait pas alors l'objet d'un rappel constant. Chez les Centraliens, au contraire, la volonté est affichée et dans les discours elle est un élément à part entière de l'identité du groupe ${ }^{49}$.

Sans rechercher la systématisation à outrance, il est possible de caractériser grossièrement le discours élaboré par chaque établissement sur les lieux qui l'abritent et sur l'architecture qui lui donne un visage : mémoriel et spontané ici, il est là programmé et démonstratif. Laissant de côté un instant le champ des représentations et de l'identité, il est tentant d'esquisser un parallèle avec les conditions et les modalités des transferts vers la banlieue accomplis par les deux institutions à la fin des années 1960. Les revues des deux écoles donnent quelques linéaments d'interprétation sur cette question. Dans Arts et Manufactures, les progrès de la construction font l'objet de compte-rendus fréquents et détaillés, les grandes étapes sont célébrées sur un ton optimiste et, pour autant qu'on puisse en juger, de l'examen des articles se dégage une image consensuelle : le

\footnotetext{
46 Arts et Manufactures, $\mathrm{n}^{\circ} 125,1962$, p. 54.

${ }^{47}$ L'énumération des noms des quatre concepteurs dans les articles consacrés aux nouveaux bâtiments n'est certes pas en soi un élément significatif du désir de présenter l'École comme une création typiquement centralienne. En revanche, tous les architectes étant morts, à l'exception de Pierre Drouin, avant l'achèvement du chantier, l'oubli presque systématique du cinquième architecte, étranger à l'établissement (il s'agit de Michel Herbert) peut éventuellement apparaittre comme un élément à prendre en compte dans l'interprétation.

48 « Pose de la première pierre de la future École centrale à Châtenay-Malabry, le 24 juin 1965 », Arts et Manufactures, n¹56, août-septembre 1965, p. 21-23.

${ }^{49}$ Le phénomène est évoqué à propos de la résidence de la rue de Citeaux. Pierre Marcilly, «La maison des élèves de l'École centrale », La Construction moderne, 44e année, $\mathrm{n}^{\circ}$ 28-29, p. 14-17. À Châtenay, la résidence est également financée par ce biais : on peut fonder une chambre pour 12000 francs. Les noms des donateurs sont sur une plaque. Arts et Manufactures, n¹93, janvier 1969, p. 57.
} 
départ est accepté par la communauté et relativement bien vécu par elle. Tout autre est le spectacle que laisse transparaître la lecture de La Jaune et la Rouge $e^{50}$ : le débat entre partisans et opposants au transfert tourne au pugilat verbal et débouche sur une véritable scission au sein de l'association, avec en ligne de mire, pour une part importante de la communauté, le projet de faire échouer l'entreprise alors même que les travaux sont en voie d'achèvement ${ }^{51}$. Le lieu du souvenir, patrimoine d'une communauté, suscite le débat passionnel; il n'en va pas de même pour la vitrine programmée de l'institution, quittée sans doute avec nostalgie, mais à laquelle on substitue sans trop de peine une autre enveloppe architecturale revêtue des attributs contemporains de la modernité.

Après la construction d'un récit des origines, l'évocation de la maison commune. Le faisceau convergent des discours sur l'identité conduit à la question essentielle : "qui sommes nous ?" L'étude des décors présents dans les établissements d'enseignement supérieur, à Polytechnique, à Centrale ou ailleurs apporte des éléments de réponse à ce problème fondamental ${ }^{52}$. Le champ des structures et des vecteurs de la personnalité propre à ces communautés est cependant plus vaste. Le discours de célébration ${ }^{53}$, l'historiographie élaborée par la communauté, la mise en place des musées institutionnels : autant de perspectives à aborder, chacune étant porteuse d'une nouvelle facette du groupe.

La richesse potentielle du chantier ouvert se devine du reste dans l'approche choisie ici : là où la monographie centrée sur le caractère propre à chaque groupe était possible et valable, le choix d'une approche croisée, l'adoption d'un point de vue comparatif fait saillir l'originalité de chaque discours. La question posée (qui sommes nous ?) se prolonge et se répercute : qui suis-je pour les autres et qui sont-ils pour moi ? Les télégrammes adressés aux Centraliens lors du centenaire de leur école laissent affleurer, sous les hommages policés et congratulations de circonstances quelques idées plus rugueuses : ainsi Polytechnique témoigne de "sa profonde amitié et de sa hante confraternitê", tout en ajoutant que "l'École polytechnique est heureuse de constater que deux anciens élèves,

\footnotetext{
${ }^{50}$ Pour la chronologie du transfert, voir : Néva Brissaud, «Le transfert à Palaiseau », Le Paris des Polytechniciens, des ingénieurs dans la ville, Paris, D.A.A.V.P., 1994, p. 45-49.

${ }^{51}$ Le transfert eut lieu cependant. Cette opération pose la question de l'appropriation des nouveaux espaces de l'École à Palaiseau. Marc Aymes, « Palaiseau, Mythologie des lieux », Universités et grandes écoles à Paris, Paris, A.A.V.P., 1999, 222 p., p. 209-212.

52 Christian Hottin, Quand la Sorbonne était peinte, étude sur les décors peints et sculptés des établissements d'enseignement supérieur parisiens, Paris, Maisonneuve et Larose, à paraître en 2000, 300 p.

${ }^{53}$ Voir à ce propos, Pierre Bourdieu, La Noblesse d'État, grandes écoles et esprit de corps, Paris, éditions de Minuit, 1989, 569 p., p. 329-330.
} 
Benoit et Olivier, étaient parmi les cinq fondateurs de l'École centrale, et que deux encore [...] y furent directeurs des études? ${ }^{54}$.

Quant à l'angle d'approche adopté - l'architecture, l'espace communautaire - on a vu que les éléments qui contribuent à la réflexion en histoire de l'architecture font partie intégrante d'un propos qui n'a pas l'histoire de l'architecture comme objet principal : maitrise d'ouvrage, maîtrise d'œuvre, modes de financement, réception des édifices, décoration des intérieurs, formation initiale des architectes. Inversement, ces problématiques sont susceptibles d'aider l'historien à s'interroger sur un concept tel que celui de "lieu de mémoire“, vidé de toute substance à force d‘être dématérialisé.

CHRISTIAN HOTTIN

Chef de la mission ethnologie Direction de l'architecture et du patrimoine Ministère de la culture Christian.hottin@culture.gouv.fr

Une version remaniée et illustrée de ce texte a été publiée dans les Livraisons d'histoire de l'architecture :

“Mythologie centralienne, mythologie polytechnicienne", Livraisons d'bistoire de l'architecture, $\mathrm{n}^{\circ} 1$, $1^{\text {er }}$ semestre 2001, 156 p., p. 61-75.

${ }^{54}$ École Centrale des Arts et Manufactures, Compte-rendu des fêtes du centenaire, 26,27, 28 et 29 mai 1929, Paris, éd. de Brunhoff, 1929, 142 p., p. 125. 